\title{
Electronic states and nature of bonding of the molecule PdGe by all electron ab initio $\mathrm{HF}-\mathrm{Cl}$ calculations and mass spectrometric equilibrium experiments
}

Shim, Irene; Kingcade, Joseph E. , Jr.; Gingerich, Karl A.

Published in:

Journal of Chemical Physics

Link to article, DOI:

$10.1063 / 1.451445$

Publication date:

1986

Document Version

Publisher's PDF, also known as Version of record

Link back to DTU Orbit

Citation (APA):

Shim, I., Kingcade, J. E. . J., \& Gingerich, K. A. (1986). Electronic states and nature of bonding of the molecule $\mathrm{PdGe}$ by all electron ab initio HF-Cl calculations and mass spectrometric equilibrium experiments. Journal of Chemical Physics, 85(11), 6629-6636. https://doi.org/10.1063/1.451445

\section{General rights}

Copyright and moral rights for the publications made accessible in the public portal are retained by the authors and/or other copyright owners and it is a condition of accessing publications that users recognise and abide by the legal requirements associated with these rights.

- Users may download and print one copy of any publication from the public portal for the purpose of private study or research.

- You may not further distribute the material or use it for any profit-making activity or commercial gain

- You may freely distribute the URL identifying the publication in the public portal 


\title{
Electronic states and nature of bonding of the molecule PdGe by all electron $a b$ initio $\mathrm{HF}-\mathrm{Cl}$ calculations and mass spectrometric equilibrium experiments
}

\author{
Irene Shim \\ Chemical Physics, Chemistry Department B. The Technical University of Denmark, DTH 301 , \\ DK-2800 Lyngby, Denmark \\ Joseph E. Kingcade, Jr. and Karl A. Gingerich \\ Department of Chemistry, Texas A\&M University, College Station, Texas 77843
}

(Received 23 July 1986; accepted 28 August 1986)

\begin{abstract}
In the present work we present all-electron ab initio Hartree-Fock (HF) and configuration interaction (CI) calculations of six electronic states of the PdGe molecule. The molecule is predicted to have a ${ }^{3} \Pi$ ground state and two low-lying excited states ${ }^{3} \Sigma^{-}$and ${ }^{1} \Sigma^{+}$. The electronic structure of the PdGe molecule has been rationalized in a simple molecular orbital picture. As part of the PdGe molecule the Pd atom essentially retains its $(4 d)^{10}$ ground term configuration. The chemical bond in the PdGe molecule has been interpreted in terms of donation and backdonation of charge. The bond is polar with a small charge transfer from the $\mathrm{Pd}$ to the Ge atom. The dissociation energy of the PdGe molecule has been determined from the mass spectrometric equilibrium data combined with the theoretical results as $D_{0}^{0}$ $=252.0 \pm 10.5 \mathrm{~kJ} \mathrm{~mol}^{-1}$.
\end{abstract}

\section{INTRODUCTION}

Investigations of the physical and chemical properties of small molecules containing transition metal atoms are of considerable current interest. ${ }^{1,2}$ In continuation of our recent work elucidating the electronic and thermodynamic properties of the first platinum metal triad carbides ${ }^{3-5}$ we have chosen to investigate the molecule PdGe. By studying the interaction between the $\mathrm{Pd}$ atom and the Ge atom we are able to explore the changes occurring in the electronic structure and nature of bonding due to the exchange of the $\mathrm{C}$ atom by the heavier group IV element $\mathrm{Ge}$. Furthermore, it is interesting to compare the chemical bond in the heteronuclear PdGe molecule as described in our theoretical work with our previous results concerning the homonuclear molecules $\mathrm{Pd}_{2}{ }^{6}$ and $\mathrm{Ge}_{2}{ }^{7,8}$

The PdGe molecule has not previously been investigated theoretically, and the experimental work concerning this molecule is limited to a brief report on the measurement of its dissociation energy. ${ }^{9}$

In the present work the PdGe molecule has been investigated by all electron ab initio Hartree-Fock (HF) and configuration interaction $(\mathrm{CI})$ calculations and also by high temperature mass spectrometric measurements. The electronic structure and the nature of bonding of the PdGe molecule have been revealed by the theoretical work, and the results obtained have been utilized to derive the dissociation energy of the molecule from the mass spectrometric data.

The HF calculations for the PdGe molecule have been carried out in the Hartree-Fock-Roothaan formalism. ${ }^{10}$ The integrals have been calculated using the program MOLECULE. ${ }^{11}$ For the HF calculations we have utilized the ALCHEMY program system, ${ }^{12}$ and the CI calculations have been performed using ALCHEMY in conjunction with the program ENERGY ${ }^{13}$ for generating the symbolic energy expressions. Finally, the oribtal plots and the deformation density map have been produced by a program especially designed for contour plotting of orbitals, densities, and electrostatic potentials. $^{14}$

\section{THEORETICAL INVESTIGATIONS}

\section{A. Basis sets and atomic calculations}

The basis sets consisted of Gaussian-type functions. For both atoms all occupied atomic orbitals except the outermost $d$ orbitals are represented by double zeta functions. The empty $5 p$ orbitals of $P d$ also have double zeta representations while the $3 d$ orbitals of $\mathrm{Ge}$ and the $4 d$ orbitals of $\mathrm{Pd}$ have been represented by triple zeta functions.

The basis set for the Pd atom has previously been described in considerable detail in connection with our work on the $\mathrm{Pd}_{2}$ molecule. ${ }^{6}$ The primitive basis $(17 s, 13 p, 8 d)$ has been contracted to $(10 s, 8 p, 5 d)$ using a segmented contraction scheme.

The basis set for the Ge atom has previously been employed in our work on the $\mathrm{Ge}_{2}$ molecule, ${ }^{7,8}$ and described in detail in Ref. 8. It has been derived by contraction of the

TABLE I. Relative energies (in a.u.) of the lowest-lying terms for the Pd and the Ge atoms. The calculated energies are results of HF calculations.

\begin{tabular}{llll}
\hline \hline Atom & Term & Calculated & Experimental $^{\mathrm{a}}$ \\
\hline $\mathrm{Pd}$ & ${ }^{1} S(4 d)^{10}$ & 0.000000 & 0.000000 \\
$\mathrm{Pd}$ & ${ }^{3} D(4 d)^{9}(5 s)^{1}$ & 0.008458 & 0.034933 \\
$\mathrm{Pd}$ & ${ }^{1} D(4 d)^{9}(5 s)^{1}$ & 0.022831 & 0.053408 \\
$\mathrm{Ge}$ & ${ }^{3} P(4 p)^{2}(4 s)^{2}$ & 0.000000 & 0.000000 \\
$\mathrm{Ge}$ & ${ }^{1} D(4 p)^{2}(4 s)^{2}$ & 0.043006 & 0.028050 \\
$\mathrm{Ge}$ & ${ }^{1} S(4 p)^{2}(4 s)^{2}$ & 0.106942 & 0.070159 \\
\hline
\end{tabular}

${ }^{a}$ Center of gravity of each multiplet has been derived from data of C. E. Moore, Natl. Bur. Std. Circ. No. 467 (U.S. GPO, Washington, D.C., 1952, 1958), Vols. 2 and 3. 
TABLE II. Total energies of the PdGe molecule as resulting from HF treatments at the internuclear distance 5.00 a.u.; also included for each state are the gross atomic charge and the total number of $d$ electrons on Pd as well as the dipole moment.

\begin{tabular}{|c|c|c|c|c|c|c|c|c|c|c|}
\hline \multirow[b]{2}{*}{ State } & \multicolumn{6}{|c|}{ Valence shell configuration } & \multirow{2}{*}{$\begin{array}{c}\text { Energy }^{\mathrm{a}} \\
\text { (a.u.) }\end{array}$} & \multirow{2}{*}{$\begin{array}{l}\text { Gross } \\
\text { atomic } \\
\text { charge }\end{array}$} & \multirow{2}{*}{$\begin{array}{c}\text { Number } \\
\text { of } \\
d \text { electrons }\end{array}$} & \multirow{2}{*}{$\begin{array}{l}\text { Dipole } \\
\text { moment } \\
\text { (D) }\end{array}$} \\
\hline & $3 \delta$ & $8 \pi$ & $9 \pi$ & $15 \sigma$ & $16 \sigma$ & $17 \sigma$ & & & & \\
\hline${ }^{3} \Pi$ & 4 & 4 & 1 & 2 & 2 & 1 & -0.040636 & -0.07 & 19.79 & 0.55 \\
\hline${ }^{3} \Sigma^{-}$ & 4 & 4 & 2 & 2 & 2 & 0 & -0.037364 & 0.13 & 19.80 & 1.81 \\
\hline${ }^{5} \Sigma^{-}$ & 4 & 4 & 2 & 2 & 1 & 1 & -0.033691 & 0.35 & 18.99 & 5.64 \\
\hline${ }^{3} \Pi, \Phi^{\mathbf{b}}$ & 3 & 4 & 1 & 2 & 2 & 2 & -0.019186 & -0.05 & 18.91 & 2.82 \\
\hline${ }^{1} \Sigma^{+}$ & 4 & 4 & 0 & 2 & 2 & 2 & -0.001577 & -0.24 & 19.68 & 0.20 \\
\hline${ }^{5} \Delta$ & 3 & 4 & 2 & 2 & 2 & 1 & 0.272019 & 0.07 & 18.92 & 3.08 \\
\hline
\end{tabular}

anergy of PdGe minus energy of the Pd and the Ge atoms, both in their ground terms.

'The wave function represents a mixture of orbital angular momenta.

most diffuse $s$ and $p$ functions in Huzinaga's basis. ${ }^{15}$ Furthermore, a diffuse $d$ function with exponent 0.223 has been added. The primitive basis $(14 s, 11 p, 6 d)$ was contracted to $(8 s, 6 p, 3 d)$.

In Table I we compare the relative energies of the lowest lying terms of the atoms Pd and Ge as derived in the HF approximation with the corresponding experimental values. It is noted that the calculated splittings are somewhat too small for Pd and too large for Ge, but more important, the sequence of the low-lying terms as derived in the HF calculations are in agreement with the experimental data.

\section{B. HF calculations on some low-lying states of the PdGe molecule}

The ground term of the $\mathrm{Pd}$ atom is ${ }^{1} S(4 d)^{10}$ and that of the Ge atom is ${ }^{3} P(4 s)^{2}(4 p)^{2}$. Coupling of the angular momenta of these two terms indicate that the ground state of the PdGe molecule should be either a ${ }^{3} \Sigma^{-}$or a ${ }^{3} \Pi$ state. Taking into account also the ${ }^{1} D(4 s)^{2}(4 p)^{2}$ first excited term of the $\mathrm{Ge}$ atom leads to the additional states ${ }^{1} \Sigma^{+},{ }^{1} \Pi$, and ${ }^{1} \Delta$, which presumably are quite low lying. However, if the first excited term of the Pd atom, ${ }^{3} D(4 d)^{9}(5 s)^{1}$, is considered then coupling of the angular momenta of this term with those of the ${ }^{3} P(4 s)^{2}(4 p)^{2}$ ground term of the Ge atom gives rise to a manifold of electronic states for the PdGe molecule, namely quintet, triplet, and singlet states of the symmetries $\Sigma^{+}, \Sigma^{-}(2), \Pi(3), \Delta(2)$, and $\Phi$, where the numbers in parenthesis show the number of states within each spin and space symmetry.

In the present work we have performed HF calculations on the states ${ }^{3} \Sigma^{-}$and ${ }^{3} \Pi$ which are the most likely ground state candidates for the PdGe molecule. Table II shows the results of the HF calculations on these states and on a few additional states at the internuclear distance 5.0 a.u. Table II contains the configuration of the valence shell of each state together with its total energy. Also included are the dipole moments as well as the total numbers of $d$ electrons and the gross atomic charges on the $\mathbf{P d}$ atom.

The numbers of $d$ electrons on the Pd atom in the individual states presented in Table II indicate that the states ${ }^{3} \mathrm{II}$, ${ }^{3} \Sigma^{-}$, and ${ }^{1} \Sigma^{+}$are indeed derivable from a Pd atom in its ${ }^{1} S(4 d){ }^{10}$ ground term while the remaining states in Table II are due to the $(4 d)^{9}(5 s)^{1}$ configuration of the $\mathrm{Pd}$ atom.
Thus, of the states investigated the lowest lying state at 5.00 a.u. is the ${ }^{3} \Pi$ state, but the ${ }^{3} \Sigma^{-}$state is only $0.09 \mathrm{eV}$ above the ${ }^{3} \Pi$ state, and at this internuclear distance both states are bound relative to the free atoms by 1.11 and $1.02 \mathrm{eV}$, respectively.

Table II also shows that the charge associated with the $\mathrm{Pd}$ atom is very much dependent on the state of the PdGe molecule considered, and even changes of its sign occur. Thus, the $P d$ atom acquires negative gross atomic charges in the ${ }^{3} \Pi$ and ${ }^{1} \Sigma^{+}$states and positive in the ${ }^{3} \Sigma^{-}$state. However, no physical significance should be attributed to the small negative charge on the Pd atom in the ${ }^{3} \Pi$ state since an examination of the signs of the dipole moments reveals that only in the case of the ${ }^{1} \Sigma^{+}$state is the negative end of the dipole vector associated with the $\mathrm{Pd}$ atom.

Table III presents the Mulliken population analyses of the three states ${ }^{3} \Pi,{ }^{3} \Sigma^{-}$, and ${ }^{1} \Sigma^{+}$that are derivable from the ground terms of the two atoms, and Fig. 1 shows contour plots of the valence orbitals of the PdGe molcule in its ${ }^{3} \Pi$ ground state. From the Mulliken population analyses as well as from the contour plots it is noted that the orbitals $16 \sigma$ and $9 \pi$ (Fig. 1) are mainly the localized $4 s$ and $4 p \pi$ orbitals of the $\mathrm{Ge}$ atom. The Mulliken population analyses indicate that the configuration of the $\mathrm{Ge}$ atom is $(4 s \sigma)^{2}(4 p \sigma)^{1}(4 p \pi)^{1}$ in the ${ }^{3} \Pi$ state of PdGe while it is $(4 s \sigma)^{2}(4 p \pi)^{2}$ and $(4 s \sigma)^{2}(4 p \sigma)^{2}$ in the ${ }^{3} \Sigma^{-}$and ${ }^{1} \Sigma^{+}$states, respectively. Taking this as a starting point the chemical bond in the ${ }^{3} \Pi$ and in the ${ }^{1} \Sigma^{+}$states can be interpreted in terms of donation and backdonation of charge. Thus, in the $8 \pi$ orbital charge is donated from the $4 d \pi$ of Pd into the $4 p \pi$ of $\mathrm{Ge}$, and in the $15 \sigma$ orbital the donation of charge is from the $4 d \sigma$ of Pd into the $4 p \sigma$ of Ge. Backdonation occurs from the $4 p \sigma$ orbital of Ge into the $5 s$ and $5 p$ orbitals of the $\mathrm{Pd}$ atom (orbital $17 \sigma$, Fig. 1) resulting in a population of $0.19 e$ in the $\mathrm{Pd} 5 s$ and of $0.09 e$ in the Pd $5 p$ orbitals for the ${ }^{3} \Pi$ state. In the case of the ${ }^{1} \Sigma^{+}$state the backdonation leads to a population of $0.44 e$ in the $\mathrm{Pd} 5 s$ orbital and to $0.12 e$ in the Pd $5 p$ orbital.

In the ${ }^{3} \Sigma^{-}$state hardly any backdonation occurs, but the donation of charge from the $4 d \sigma$ orbital of Pd into the $4 p \sigma$ orbital of Ge results in a total positive charge of $0.13 e$ on the $\mathrm{Pd}$ atom. This is considerably less than the $0.28 e$ found in the ${ }^{3} \Sigma^{-}$ground state of the PdC molecule, ${ }^{3}$ but this is 
TABLE III. Mulliken population analyses of the valence orbitals of the PdGe molecule in the ${ }^{3} \Pi$ ground state and in the lowest lying excited states ${ }^{3} \Sigma^{-}$and ${ }^{1} \Sigma^{+}$. The wave functions have been derived in HF calculations at the internuclear distance 5.0 a.u.

\begin{tabular}{|c|c|c|c|c|c|c|c|c|c|c|c|}
\hline \multirow[b]{3}{*}{ State } & \multirow[b]{3}{*}{ Orbital } & & & \multirow{3}{*}{$\begin{array}{c}\text { Overlap } \\
\text { population }\end{array}$} & \multicolumn{6}{|c|}{ Orbital analyses } & \multirow{3}{*}{$\begin{array}{l}\text { Occupation } \\
\text { number }\end{array}$} \\
\hline & & \multicolumn{2}{|c|}{$\begin{array}{l}\text { Atomic } \\
\text { population }\end{array}$} & & \multicolumn{3}{|c|}{ Pd } & \multicolumn{3}{|c|}{$\mathrm{Ge}$} & \\
\hline & & $\mathbf{P d}$ & $\mathrm{Ge}$ & & $s$ & $p$ & $d$ & $s$ & $p$ & $d$ & \\
\hline \multirow[t]{6}{*}{${ }^{3} n$} & $15 \sigma$ & 1.81 & 0.14 & 0.05 & 0.03 & 0.01 & 1.79 & 0.04 & 0.12 & 0.01 & 2.00 \\
\hline & $16 \sigma$ & 0.06 & 1.87 & 0.07 & 0.02 & 0.01 & 0.07 & 1.91 & 0.00 & 0.00 & 2.00 \\
\hline & $17 \sigma$ & 0.17 & 0.72 & 0.11 & 0.15 & 0.05 & 0.03 & 0.00 & 0.78 & 0.00 & 1.00 \\
\hline & $8 \pi$ & 3.83 & 0.06 & 0.10 & 0.00 & 0.00 & 3.88 & 0.00 & 0.09 & 0.02 & 4.00 \\
\hline & $9 \pi$ & 0.04 & 0.96 & 0.00 & 0.00 & 0.02 & 0.02 & 0.00 & 0.96 & 0.00 & 1.00 \\
\hline & $3 \delta$ & 4.00 & 0.00 & 0.00 & 0.00 & 0.00 & 4.00 & 0.00 & 0.00 & 0.00 & 4.00 \\
\hline Total & & 45.90 & 31.76 & 0.35 & 8.19 & 18.09 & 19.79 & 7.94 & 13.95 & 10.04 & \\
\hline \multirow[t]{5}{*}{${ }^{3} \Sigma^{-}$} & $15 \sigma$ & 1.70 & 0.23 & 0.07 & 0.01 & 0.02 & 1.71 & 0.11 & 0.14 & 0.01 & 2.00 \\
\hline & $16 \sigma$ & 0.11 & 1.83 & 0.07 & 0.01 & 0.00 & 0.13 & 1.86 & 0.00 & 0.00 & 2.00 \\
\hline & $8 \pi$ & 3.90 & 0.03 & 0.07 & 0.00 & 0.00 & 3.94 & 0.00 & 0.04 & 0.02 & 4.00 \\
\hline & $9 \pi$ & 0.05 & 1.93 & 0.02 & 0.00 & 0.04 & 0.02 & 0.00 & 1.94 & 0.00 & 2.00 \\
\hline & $3 \delta$ & 4.00 & 0.00 & 0.00 & 0.00 & 0.00 & 4.00 & 0.00 & 0.00 & 0.00 & 4.00 \\
\hline Total & & 45.76 & 32.01 & 0.23 & 8.02 & 18.05 & 19.80 & 7.97 & 14.13 & 10.03 & \\
\hline \multirow[t]{5}{*}{${ }^{\prime} \Sigma^{+}$} & $15 \sigma$ & 1.54 & 0.40 & 0.06 & 0.00 & -0.01 & 1.58 & 0.03 & 0.39 & 0.01 & 2.00 \\
\hline & $16 \sigma$ & 0.04 & 1.86 & 0.10 & 0.03 & 0.02 & 0.05 & 1.91 & 0.00 & 0.00 & 2.00 \\
\hline & $17 \sigma$ & 0.69 & 1.14 & 0.18 & 0.41 & 0.11 & 0.25 & -.02 & 1.25 & 0.00 & 2.00 \\
\hline & $8 \pi$ & 3.74 & 0.12 & 0.14 & 0.00 & 0.00 & 3.80 & 0.00 & 0.16 & 0.03 & 4.00 \\
\hline & $3 \delta$ & 4.00 & 0.00 & 0.00 & 0.00 & 0.00 & 4.00 & 0.00 & 0.00 & 0.00 & 4.00 \\
\hline Total & & 46.00 & 31.52 & 0.49 & 8.44 & 18.12 & 19.68 & 7.92 & 13.80 & 10.04 & \\
\hline
\end{tabular}

consistent with the higher electronegativity of the $\mathrm{C}$ atom relative to the Ge atom.

\section{Results of $\mathrm{Cl}$ calculations on the PdGe molecule}

We have performed CI calculations on the PdGe molecule allowing full reorganization within the valence shells of the molecule, thus ensuring that the wave functions are appropriate for describing the molecule in the dissociation limit. The calculations have been carried out in the subgroup $C_{2 v}$ of the full symmetry group $C_{\infty v}$ of the molecule. The number of configurations included within each symmetry species of $C_{2 v}$ reaches 36 for the quintet states, 164 for the triplet states, and 152 for the singlet states.

Analogous to our work on the carbide molecule, $\mathrm{RhC}^{4}$ we intended to describe the low-lying states of the PdGe molecule by performing $\mathrm{CI}$ calculations using just one set of molecular orbitals. In order to identify an appropriate set of molecular oribtals we carried out CI calculations utilizing the different sets of orbitals that had been optimized in the HF calculations presented in Table II. The results of these calculations revealed that it was not possible to describe the electronic states of the PdGe molecule arising from the $(4 d)^{10}$ as well as from the $(4 d)^{9}(5 s)^{1}$ configuration of the $\mathrm{Pd}$ atom with sufficient accuracy using only one set of molecular orbitals. Therefore, we finally decided to investigate only the states originating from the $(4 d)^{10}$ configuration of the Pd atom. For this purpose we have chosen to utilize the molecular oribtals optimized for the ${ }^{3} \Pi$ state since these orbitals resulted in the lowest energies for the ${ }^{3} \Pi$ ground state as well as for the ${ }^{3} \Sigma^{-}$first excited state at the internuclear distance 5.00 a.u.

The molecular orbitals have been optimized for the ${ }^{3} \Pi$ state at the internuclear distances 3.6, 4.2, 5.0, 5.5, and 6.2 a.u. and utilized in the CI calculations described above. The potential energies obtained in these calculations have been fitted to Morse curves, and in Fig. 2 we present the potential energy curves of the six electronic states of the PdGe molecule arising from interaction between the $\mathrm{Pd}$ atom in the $(4 d)^{10}$ configuration and the Ge atom in the $(4 s)^{2}(4 p)^{2}$ configuration. The equilibrium distances, the vibrational frequencies, and the transition energies derived in the fitting process are reported in Table IV together with the similar results derived on basis of the HF calculations. Also included are the dissociation energies determined as differences between the energies of the PdGe molecule at the equilibrium distance and the sum of the HF energies of the atoms. This procedure is reasonable even for the results of the CI calculations, because the CI wave functions include only molecular correlation that vanish in the dissociation limit.

Table $\mathrm{V}$ shows the occupations of the natural valence orbitals as functions of the internuclear distance for the three lowest lying states ${ }^{3} \Pi,{ }^{3} \Sigma^{-}$, and ${ }^{1} \Sigma^{+}$. In Table VI we present the Mulliken population analyses of the CI wave functions for these three states at the internuclear distance 5.00 a.u.

From Table IV it is noted that both in the HF and in the CI calculations the predicted ground state of the PdGe molecule is a ${ }^{3} \Pi$ state with a ${ }^{3} \Sigma^{-}$state as the first exited state. A similar sequence of the two lowest lying states has also been found for the PdSi molecule, while an interchange of these states occurs for the PdC molecule. ${ }^{16}$ The sequence of the low-lying electronic states for these Pd-group IV diatomics can be rationalized on the basis of a simple molecular orbital picture involving only the partly occupied $p \sigma$ and $p \pi$ orbitals of the group IV elements. The $p \sigma$ and $p \pi$ orbitals should be 

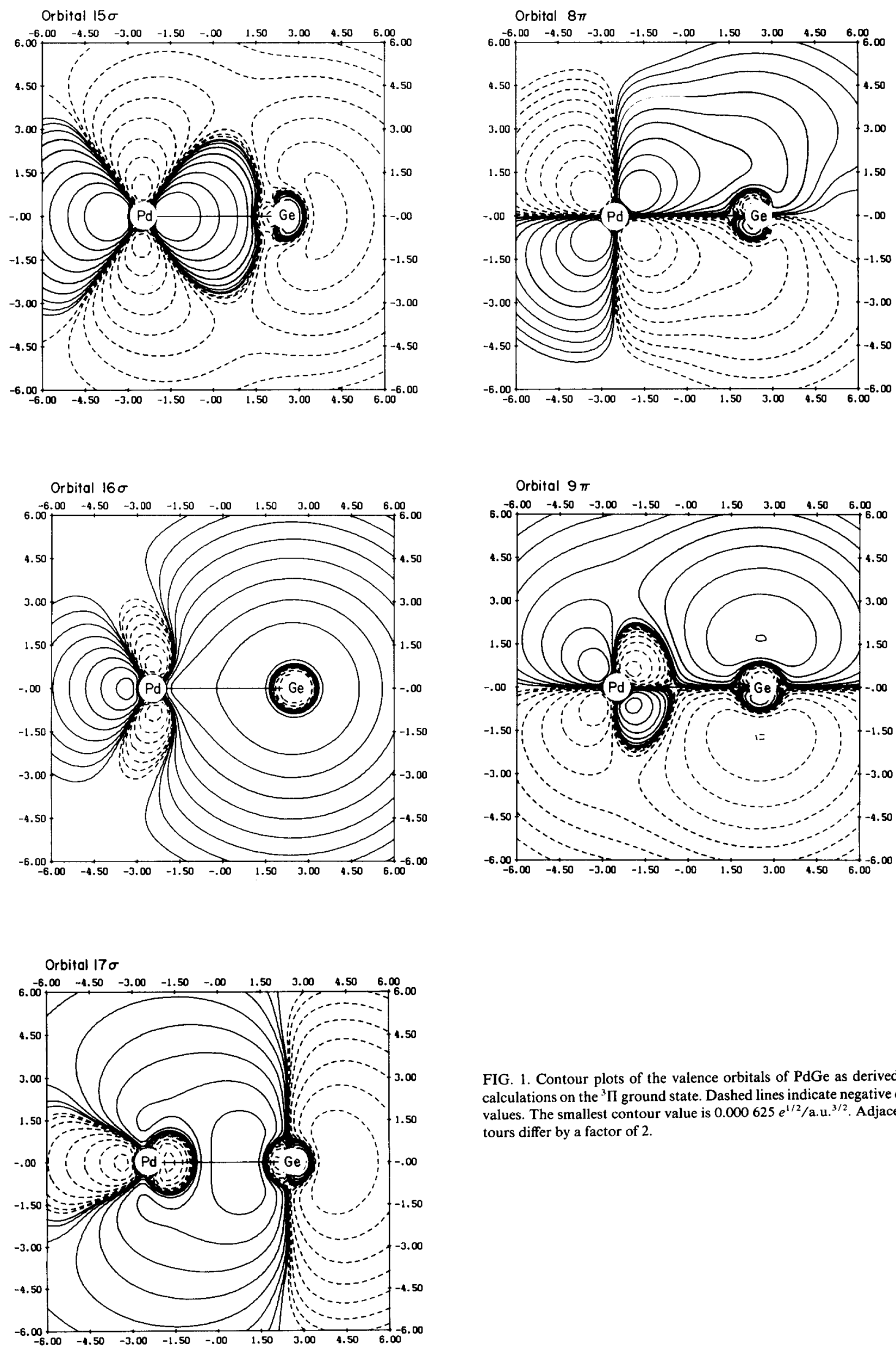

FIG. 1. Contour plots of the valence orbitals of PdGe as derived in HF calculations on the ${ }^{3} \Pi$ ground state. Dashed lines indicate negative contour values. The smallest contour value is $0.000625 e^{1 / 2} /$ a.u..$^{3 / 2}$. Adjacent contours differ by a factor of 2 . 
$E($ a.u. $)+7012$ a.u.



FIG. 2. Potential energy curves for six electronic states of the PdGe molecule as derived in CI calculations.

close in energy, since the energy splittings between the ${ }^{3} \Pi(\mathrm{p} \sigma)^{1}(p \pi)^{1}$ and the ${ }^{3} \Sigma^{-}(p \pi)^{2}$ states is only $0.28 \mathrm{eV}$ for PdGe. It is noted that the energy splitting between these states arising from different orbital configurations is smaller than the exchange energies due to the different exchange couplings of the angular momenta within each orbital configuration. Compared to the energy splitting of $0.28 \mathrm{eV}$ between the ${ }^{3} \Pi(p \sigma)^{1}\left(p \pi^{1}\right)$ and the ${ }^{3} \Sigma^{-}(p \pi)^{2}$ states, the energy splitting is $0.92 \mathrm{eV}$ between the ${ }^{3} \Pi$ and ${ }^{1} \Pi$ states both arising from the orbital configuration $(p \sigma)^{1}(p \pi)^{1}$, and it is $1.12 \mathrm{eV}$ between the states ${ }^{3} \Sigma^{-}$and ${ }^{1} \Delta$ both with orbital configuration $(p \pi)^{2}$.

TABLE IV. Spectroscopic constants for the low-lying electronic states of the $\mathrm{PdGe}$ molecule as derived in $\mathrm{HF}$ and $\mathrm{CI}$ calculations.

\begin{tabular}{|c|c|c|c|c|}
\hline State & $\begin{array}{c}\text { Equilibrium } \\
\text { distance (a.u.) }\end{array}$ & $\begin{array}{c}\text { Transition } \\
\text { energy }(\mathrm{eV})\end{array}$ & $\begin{array}{l}\text { Vibrational } \\
\text { frequency } \\
\left(\mathrm{cm}^{-1}\right)\end{array}$ & $\begin{array}{l}\text { Dissociation } \\
\text { energy (eV) }\end{array}$ \\
\hline${ }^{3} \Pi$ & 4.59 & 0.00 & 180 & 1.19 \\
\hline${ }^{3} \Sigma^{-}, \quad$ HF & 4.73 & 0.14 & 154 & 1.05 \\
\hline${ }^{5} \Sigma^{-}, \quad H F$ & 5.09 & 0.27 & 147 & 0.92 \\
\hline${ }^{\prime} \Sigma^{+}, \quad$ HF & 4.35 & 0.78 & 265 & 0.41 \\
\hline${ }^{3} \Pi$ & 4.47 & 0.00 & 209 & 1.60 \\
\hline${ }^{3} \Sigma^{-}, \quad$ CI & 4.62 & 0.28 & 174 & 1.32 \\
\hline $1^{1} \Sigma^{+}, \mathrm{CI}$ & 4.28 & 0.46 & 288 & 1.14 \\
\hline 'II, & 4.44 & 0.92 & 230 & 0.68 \\
\hline${ }^{1} \Delta, \quad$ CI & 4.54 & 1.40 & 184 & 0.20 \\
\hline $2{ }^{1} \Sigma^{+}, \mathrm{CI}$ & 4.49 & 2.07 & 210 & -0.48 \\
\hline
\end{tabular}

TABLE V. Occupation of the natural valence orbitals of the ${ }^{3} \Pi$ ground state and of the two lowest excited states ${ }^{3} \Sigma^{-}$and ${ }^{1} \Sigma^{+}$of PdGe as functions of the internuclear distance.

\begin{tabular}{|c|c|c|c|c|c|c|}
\hline \multirow[b]{3}{*}{ State } & \multirow[b]{3}{*}{ Orbital } & \multicolumn{5}{|c|}{ Occupation } \\
\hline & & \multicolumn{5}{|c|}{ Distance (a.u.) } \\
\hline & & 3.6 & 4.2 & 5.0 & 5.5 & 6.2 \\
\hline \multirow[t]{6}{*}{${ }^{3} \Pi$} & $15 \sigma$ & 2.00 & 2.00 & 2.00 & 2.00 & 2.00 \\
\hline & $16 \sigma$ & 1.97 & 1.97 & 1.97 & 1.97 & 1.97 \\
\hline & $17 \sigma$ & 1.01 & 1.01 & 1.00 & 1.00 & 1.00 \\
\hline & $8 \pi$ & 3.97 & 3.97 & 3.99 & 4.00 & 4.00 \\
\hline & $9 \pi$ & 1.05 & 1.05 & 1.04 & 1.03 & 1.03 \\
\hline & $3 \delta$ & 4.00 & 4.00 & 4.00 & 4.00 & 4.00 \\
\hline \multirow[t]{6}{*}{${ }^{3} \Sigma^{-}$} & $15 \sigma$ & 2.00 & 2.00 & 1.99 & 2.00 & 2.00 \\
\hline & $16 \sigma$ & 1.96 & 1.95 & 1.96 & 1.97 & 1.97 \\
\hline & $17 \sigma$ & 0.05 & 0.06 & 0.05 & 0.03 & 0.03 \\
\hline & $8 \pi$ & 3.99 & 3.99 & 4.00 & 4.00 & 4.00 \\
\hline & $9 \pi$ & 2.01 & 2.01 & 2.00 & 2.00 & 2.00 \\
\hline & $3 \delta$ & 4.00 & 4.00 & 4.00 & 4.00 & 4.00 \\
\hline \multirow[t]{6}{*}{${ }^{1} \Sigma^{+}$} & $15 \sigma$ & 2.00 & 2.00 & 2.00 & 2.00 & 2.00 \\
\hline & $16 \sigma$ & 1.98 & 1.97 & 1.97 & 1.97 & 1.97 \\
\hline & $17 \sigma$ & 1.91 & 1.93 & 1.83 & 1.70 & 1.57 \\
\hline & $8 \pi$ & 3.95 & 3.94 & 3.98 & 4.00 & 4.00 \\
\hline & $9 \pi$ & 0.16 & 0.16 & 0.22 & 0.33 & 0.47 \\
\hline & $3 \delta$ & 4.00 & 4.00 & 4.00 & 4.00 & 4.00 \\
\hline
\end{tabular}

In the molecular orbital picture associated with the molecules PdGe and PdSi the $p \sigma$ orbital should have slightly lower energy than the $p \pi$ orbital. For the PdC molecule an interchange of the orbitals occurs so the $p \pi$ orbital has lower energy than the $p \sigma$ orbital. These alterations of the sequences of the molecular orbitals are also consistent with the electronic ground states of the homonuclear group IV diatomics. Thus, the ground state of $C_{2}$ is ${ }^{1} \Sigma_{g}+\left(2 p \pi_{u}\right)^{4},{ }^{17}$ and that of $\mathrm{Ge}_{2}$ is ${ }^{3} \Sigma_{g}^{-}\left(4 p \sigma_{g}\right)^{2}\left(4 p \pi_{u}\right)^{27,8,18}$ while the ground state of $\mathrm{Si}_{2}$ presumably is ${ }^{3} \Sigma_{g}^{-}\left(3 p \sigma_{g}\right)^{2}\left(3 p \pi_{u}\right)^{2}$ with the ${ }^{3} \Pi_{u}\left(3 p \sigma_{g}\right)^{1}\left(3 p \pi_{u}\right)^{3}$ state at very low energy. ${ }^{3}$ A similar comparison with the chemical bond in the $\mathrm{Pd}_{2}$ molecule ${ }^{6}$ is not as instructive, because the bond in the $\mathrm{Pd}_{2}$ molecule is formed between two $\mathrm{Pd}$ atoms with the $(4 d)^{9}(5 s)^{1}$ configuration. In the Pd-group IV diatomics the Pd atom essentially retains its $(4 d)^{10}$ ground term configuration.

The occupations of the natural orbitals presented in Table $V$ indicate that for the ${ }^{3} \Pi$ and ${ }^{3} \Sigma^{-}$states only minor changes of the valence shell configurations occur at internuclear distances in between 3.6 and 6.2 a.u. In the ${ }^{1} \Sigma^{+}$state the major configuration is $(p \sigma)^{2}$. This configuration contribute $89 \%$ to the wave function at 4.2 a.u., but only $77 \%$ at $6.2 \mathrm{a}$.u. due to an increasing admixture of the $(p \pi)^{2}$ configuration.

The Mulliken population analyses of the $\mathrm{CI}$ wave functions for the ${ }^{3} \Pi,{ }^{3} \Sigma^{-}$, and ${ }^{1} \Sigma^{+}$states (Table VI) all resemble the Mulliken population analyses of the HF wave functions (Table III) closely. This indicates that it is adequate to utilize the molecular orbitals optimized for the ${ }^{3} \Pi$ state to describe all three states as has been done in the present work. Furthermore, the interpretation of the chemical bond of the PdGe molecule in terms of donation and backdonation of charge as derived from the HF calculations is also adequate 
TABLE VI. Mulliken population analyses of the valence orbitals of the PdGe molecule in the ${ }^{3} \Pi_{\text {ground }}$ state and in the lowest lying excited states ${ }^{3} \Sigma^{-}$and ${ }^{\prime} \Sigma^{+}$. The wave functions have been derived in $\mathrm{CI}$ calculations at the internuclear distance 5.0 a.u

\begin{tabular}{|c|c|c|c|c|c|c|c|c|c|c|c|}
\hline \multirow[b]{3}{*}{ State } & \multirow[b]{3}{*}{ Orbital } & & & \multirow{3}{*}{$\begin{array}{c}\text { Overlap } \\
\text { population }\end{array}$} & \multicolumn{6}{|c|}{ Oribtal analyses } & \multirow{3}{*}{$\begin{array}{c}\text { Occupation } \\
\text { number }\end{array}$} \\
\hline & & \multicolumn{2}{|c|}{$\begin{array}{c}\text { Atomic } \\
\text { population }\end{array}$} & & \multicolumn{3}{|c|}{ Pd } & \multicolumn{3}{|c|}{$\mathrm{Ge}$} & \\
\hline & & $\mathbf{P d}$ & $\mathrm{Ge}$ & & $s$ & $p$ & $d$ & $s$ & $p$ & $d$ & \\
\hline \multirow[t]{6}{*}{${ }^{3} \Pi$} & $15 \sigma$ & 1.73 & 0.24 & 0.03 & -0.01 & -0.02 & 1.78 & 0.12 & 0.12 & 0.01 & 2.00 \\
\hline & $16 \sigma$ & 0.11 & 1.76 & 0.10 & 0.05 & 0.04 & 0.07 & 1.80 & 0.01 & 0.00 & 1.97 \\
\hline & $17 \sigma$ & 0.18 & 0.71 & 0.11 & 0.15 & 0.05 & 0.03 & 0.00 & 0.77 & 0.00 & 1.00 \\
\hline & $8 \pi$ & 3.79 & 0.08 & 0.11 & 0.00 & 0.00 & 3.85 & 0.00 & 0.12 & 0.02 & 3.99 \\
\hline & $9 \pi$ & 0.04 & 1.00 & 0.00 & 0.00 & 0.02 & 0.02 & 0.00 & 1.00 & 0.00 & 1.04 \\
\hline & $3 \delta$ & 4.00 & 0.00 & 0.00 & 0.00 & 0.00 & 4.00 & 0.00 & 0.00 & 0.00 & 4.00 \\
\hline Total & & 45.85 & 31.80 & 0.36 & 8.19 & 18.09 & 19.75 & 7.92 & 14.02 & 10.04 & \\
\hline \multirow[t]{6}{*}{${ }^{3} \Sigma^{-}$} & $15 \sigma$ & 1.20 & 0.75 & 0.04 & -0.03 & -0.04 & 1.29 & 0.65 & 0.11 & 0.01 & 1.99 \\
\hline & $16 \sigma$ & 0.60 & 1.27 & 0.09 & 0.07 & 0.05 & 0.52 & 1.26 & 0.05 & 0.00 & 1.96 \\
\hline & $17 \sigma$ & 0.01 & 0.03 & 0.00 & 0.01 & 0.00 & 0.00 & 0.00 & 0.03 & 0.00 & 0.05 \\
\hline & $8 \pi$ & 3.87 & 0.04 & 0.09 & 0.00 & 0.00 & 3.92 & 0.00 & 0.05 & 0.02 & 4.00 \\
\hline & $9 \pi$ & 0.05 & 1.94 & 0.01 & 0.00 & 0.03 & 0.02 & 0.00 & 1.94 & 0.00 & 2.00 \\
\hline & $3 \delta$ & 4.00 & 0.00 & 0.00 & 0.00 & 0.00 & 4.00 & 0.00 & 0.00 & 0.00 & 4.00 \\
\hline Total & & 45.73 & 32.02 & 0.24 & 8.05 & 18.05 & 19.76 & 7.92 & 14.19 & 10.04 & \\
\hline \multirow[t]{6}{*}{${ }^{\prime} \Sigma^{+}$} & $15 \sigma$ & 1.85 & 0.14 & 0.02 & 0.00 & -0.01 & 1.86 & 0.04 & 0.09 & 0.01 & 2.00 \\
\hline & $16 \sigma$ & 0.06 & 1.78 & 0.13 & 0.06 & 0.04 & 0.02 & 1.85 & 0.00 & 0.00 & 1.97 \\
\hline & $17 \sigma$ & 0.27 & 1.37 & 0.18 & 0.25 & 0.09 & 0.03 & 0.02 & 1.44 & 0.00 & 1.83 \\
\hline & $8 \pi$ & 3.68 & 0.16 & 0.14 & 0.00 & 0.00 & 3.75 & 0.00 & 0.21 & 0.02 & 3.98 \\
\hline & $9 \pi$ & 0.02 & 0.21 & 0.00 & 0.00 & 0.00 & 0.01 & 0.00 & 0.20 & 0.00 & 0.22 \\
\hline & $3 \delta$ & 4.00 & 0.00 & 0.00 & 0.00 & 0.00 & 4.00 & 0.00 & 0.00 & 0.00 & 4.00 \\
\hline Total & & 45.87 & 31.66 & 0.48 & 8.32 & 18.12 & 19.67 & 7.91 & 13.95 & 10.04 & \\
\hline
\end{tabular}

when considering the results of the CI calculations.

For the ${ }^{3} \Pi$ ground state the dipole moment at the internuclear distance 5.00 a.u. increases from $0.55 \mathrm{D}$ in the $\mathrm{HF}$ approximation to $1.08 \mathrm{D}$ in the $\mathrm{CI}$ approximation. In both approximations the Mulliken population analyses of the

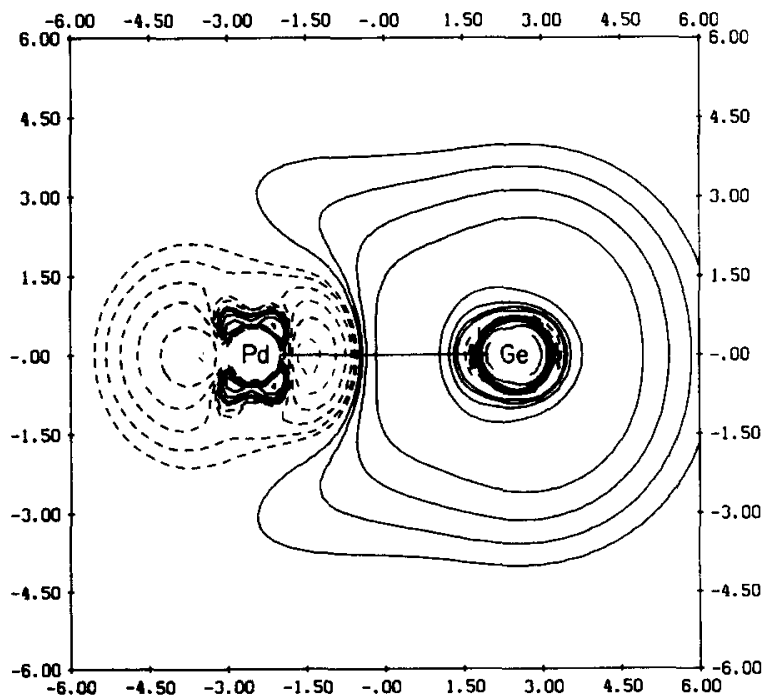

FIG. 3. Deformation density map for the ${ }^{3} \Pi$ ground state of the PdGe molecule at the internuclear distance 5.00 a.u. The superpositioned atomic charge densities from the $(4 d)^{10}$ configuration of Pd and the $(4 s)^{2}(4 p)^{2}$ configuration of $\mathrm{Ge}$ has been subtracted from the molecular charge density. Solid contours show enhanced electron charge relative to the superpositioned atoms, dashed contours show diminished charge. The smallest contour value is $0.000625 \mathrm{e} / \mathrm{a} \cdot \mathrm{u}{ }^{3}$. Adjacent contours differ by a factor of 2 . The enhancement of the negative charge at the $\mathrm{Ge}$ atom reaches a maximum value in between 0.005 and 0.010 e/a.u. ${ }^{3}$. wave functions indicate that the $\mathrm{Pd}$ atom acquires a small negative charge, but this has no physical significance, since the negative end of the dipole vector in both cases is associated with the Ge atom.

Figure 3 shows a deformation density map of the PdGe molecule at the internuclear distance 5.00 a.u. It has been derived by subtracting the spherical averaged, superpositioned atomic charge densities from the charge density of the molecule as determined from the $\mathrm{CI}$ wave function of its ${ }^{3} \mathrm{II}$ ground state. It appears that the chemical bond between the $\mathrm{Pd}$ and the $\mathrm{Ge}$ atoms is essentially due to a transfer of charge from the Pd to the Ge atom in agreement with the direction of the dipole vector.

\section{MASS SPECTROMETRIC MEASUREMENTS}

The mass spectrometer used in the present investigation is a single-focusing instrument coupled with a Knudsen effusion cell. The Knudsen cell was machined from $1 / 2$ in. solid tantalum rod with an 0.040 in. diam orifice. The sample charge of $190 \mathrm{mg}$ palladium powder, $200 \mathrm{mg}$ germanium powder, and $17 \mathrm{mg}$ silver wire was contained in a graphite crucible. The sample and crucible were then placed inside the tantalum cell. The molecular beam effusing from the Knudsen cell into the ion source region was ionized with 20 $\mathrm{V}$ electrons. The electron emission current was maintained at $0.4 \mathrm{~mA}$ throughout the entire run.

The positively charged ionic species formed were identified by their mass-to-charge ratio $(m / e)$, shutter effect, and ionization efficiency curves. Isotopic intensity distributions were obtained to identify the monatomic and diatomic species. The measured total multiplier anode currents for the 
TABLE VII. Measured total ion intensities used in the evaluation of the reaction enthalpies involving $\operatorname{PdGe}(g)$.

\begin{tabular}{ccccc}
\hline \hline & & \multicolumn{2}{c}{ Ion intensities (in amp) } & \\
\cline { 2 - 5 } Temp $(\mathrm{K})$ & $\mathrm{Ge}^{+}$ & $\mathrm{Ge}_{2}^{+}$ & $\mathrm{Pd}^{+}$ & $\mathrm{PdGe}^{+}$ \\
\hline 1744 & $2.395(-07)^{\mathrm{a}}$ & $2.267(-09)$ & $3.402(-10)$ & $7.579(-12)$ \\
1778 & $3.583(-07)$ & $3.224(-09)$ & $6.769(-10)$ & $2.064(-11)$ \\
1810 & $5.153(-07)$ & $5.012(-09)$ & $1.079(-09)$ & $3.294(-12)$ \\
1595 & $3.036(-08)$ & $1.830(-10)$ & $3.659(-11)$ & $6.058(-13)$ \\
1635 & $5.645(-08)$ & $3.765(-10)$ & $7.647(-11)$ & $1.049(-12)$ \\
1670 & $9.029(-08)$ & $6.624(-10)$ & $1.233(-10)$ & $2.915(-12)$ \\
1661 & $7.287(-08)$ & $4.785(-10)$ & $9.440(-11)$ & $1.603(-12)$ \\
1704 & $1.416(-07)$ & $1.100(-09)$ & $2.239(-10)$ & $5.749(-12)$ \\
1748 & $2.549(-07)$ & $2.149(-09)$ & $4.621(-10)$ & $1.040(-11)$ \\
1783 & $3.783(-07)$ & $3.328(-09)$ & $8.573(-10)$ & $2.663(-11)$ \\
1841 & $6.827(-07)$ & $5.855(-09)$ & $1.986(-09)$ & $5.358(-11)$ \\
1826 & $5.684(-07)$ & $4.760(-09)$ & $1.672(-09)$ & $3.944(-11)$ \\
1796 & $3.851(-07)$ & $3.131(-09)$ & $1.061(-09)$ & $2.216(-11)$ \\
1659 & $5.473(-08)$ & $3.312(-10)$ & $2.065(-10)$ & $2.880(-12)$ \\
1694 & $1.021(-07)$ & $6.498(-10)$ & $2.005(-10)$ & $4.001(-12)$ \\
1783 & $3.107(-07)$ & $2.443(-09)$ & $8.452(-10)$ & $1.312(-11)$ \\
1822 & $4.726(-07)$ & $3.723(-09)$ & $1.500(-09)$ & $3.338(-11)$ \\
1855 & $6.658(-07)$ & $5.188(-09)$ & $2.887(-09)$ & $5.699(-11)$ \\
1903 & $9.794(-07)$ & $6.789(-09)$ & $6.147(-09)$ & $1.720(-10)$ \\
1946 & $1.394(-06)$ & $8.539(-09)$ & $1.226(-08)$ & $1.905(-10)$ \\
\hline \hline
\end{tabular}

"Numbers in parentheses represent powers of ten.

pertinent species are listed in Table VII. The Gibbs energy functions $-\left(G_{T}^{0}-H_{298}^{0}\right) / T$, and the heat content functions $H_{T}^{0}-H_{298}^{0}$ needed in the evaluation of the reaction enthalpies were taken from literature ${ }^{19}$ for the atomic species $P d(g)$ and $\mathrm{Ge}(\mathrm{g})$. The Gibbs energy functions and heat content functions for the observed molecule $\mathrm{Ge}_{2}(\mathrm{~g})$ were taken the same as those employed in Kingcade et al. ${ }^{8}$ For $\operatorname{PdGe}(\mathrm{g})$ the thermal functions were computed according to statistical thermodynamic procedures using the harmonic oscillator, rigid rotator approximation, ${ }^{20}$ and the molecular constants derived in the present theoretical investigation. At the respective temperatures $298,1500,1700,1900$, and 2100 $\mathrm{K}$ the derived Gibbs energy functions $-\left(G_{T}^{0}-H_{0}^{0}\right) / T$ are $237.8,295.7,300.5,304.7$, and $308.6 \mathrm{~kJ} \mathrm{~mol}^{-1} \mathrm{~K}^{-1}$, and the heat content functions $H_{T}^{0}-H_{0}^{0}$, are $10.11,56.57,64.56$, 72.58 , and $80.59 \mathrm{~kJ} \mathrm{~mol}^{-1}$.

The measured ion intensities $I_{i}$ (Table VII) were related to the corresponding partial pressures $p_{i}$ according to the relation $p_{i}=k_{i} I_{i} T$. The calibration constant $k_{i}$ has been determined using the known $\mathbf{A g}_{2}=2 \mathrm{Ag}$ equilibrium. The values in atm $\mathrm{A}^{-1} \mathrm{~K}^{-1}$ are $\mathrm{Ag}, 1.55 \pm 0.15 ; \mathrm{Ge}, 1.16 ; \mathrm{Ge}_{2}$, 0.97; Pd, 1.36; and PdGe, 0.86.
The gas phase equilibrium reactions considered in the evaluation of the dissociation energy of $\operatorname{PdGe}(g)$ are

$$
\mathrm{PdGe}=\mathrm{Pd}+\mathrm{Ge}
$$

and

$\mathrm{PdGe}+\mathrm{Ge}=\mathrm{Pd}+\mathrm{Ge}_{2}$.

The second-law enthalpy changes, $\Delta H_{T}^{0}$, for reactions (1) and (2) were obtained from the $\log K_{p}$ vs $1 / T$ plot as $\Delta H_{1753}^{0}=279.6 \pm 12.3 \mathrm{~kJ} \mathrm{~mol}^{-1}$ for reaction (1), and $\Delta H_{1753}^{0}=-9.5 \pm 12.2 \mathrm{~kJ} \mathrm{~mol}^{-1}$ for reaction (2). The respective $\Delta H_{0}^{0}$ values are 261.1 and $-2.2 \mathrm{~kJ} \mathrm{~mol}^{-1}$.

The third-law evaluation for the same reactions yielded an enthalpy $\Delta H_{0}^{o}$ of $244.4 \pm 5.7$ and $16.3 \pm 5.4 \mathrm{~kJ} \mathrm{~mol}^{-1}$ for reactions (1) and (2), respectively. The error terms given represent standard deviations.

The evaluated second-law entropy $\Delta S_{298}^{0}$ for reaction (1) was $72.0 \pm 7.0 \mathrm{~J} \mathrm{~K}^{-1} \mathrm{~mol}^{-1}$ and for reaction (2), $-1.7 \pm 6.9 \mathrm{~J} \mathrm{~K}^{-1} \mathrm{~mol}^{-1}$. The corresponding third-law entropies are $63.0 \mathrm{~J} \mathrm{~K}^{-1} \mathrm{~mol}^{-1}$ and $-9.2 \mathrm{~J} \mathrm{~K}^{-1} \mathrm{~mol}^{-1}$, respectively.

The dissociation energy of PdGe was derived as $D_{0}^{0}$ $=252.8 \pm 10.5\left(D_{298}^{0}=256.2\right) \mathrm{kJ} \mathrm{mol}^{-1}$ from reaction

TABLE VIII. Summary of the reaction enthalpies and entropies for the gaseous molecule PdGe.

\begin{tabular}{|c|c|c|c|c|c|c|c|}
\hline \multirow[b]{2}{*}{ Reaction } & \multirow{2}{*}{$\begin{array}{c}\text { Temp. range (K) } \\
\text { (No. of data) }\end{array}$} & \multirow{2}{*}{$\begin{array}{c}\Delta H_{T}^{0} \\
\left(\mathrm{~kJ} \mathrm{~mol}^{-1}\right)\end{array}$} & \multicolumn{2}{|c|}{$\Delta H_{0}^{0}\left(\mathrm{~kJ} \mathrm{~mol}^{-1}\right)$} & \multicolumn{2}{|c|}{$\Delta S_{298}^{0}\left(\mathrm{~J} \mathrm{~K}^{-1} \mathrm{~mol}^{-1}\right)$} & \multirow{2}{*}{$\begin{array}{c}\text { Average } \\
D_{0}^{o}\end{array}$} \\
\hline & & & Second law & Third law & Second law & Third law & \\
\hline $\mathrm{PdGe}=\mathrm{Pd}+\mathrm{Ge}$ & $\begin{array}{c}1595-1946 \\
(19)\end{array}$ & $279.6 \pm 12.3$ & 261.1 & $244.4 \pm 5.7$ & $72.0 \pm 7.0$ & 63.0 & 252.8 \\
\hline \multirow[t]{2}{*}{$\mathrm{PdGe}+\mathrm{Ge}=\mathrm{Pd}+\mathrm{Ge}_{2}$} & $\begin{array}{l}1596-1946 \\
(19)\end{array}$ & $-9.5 \pm 12.2$ & -2.2 & $-16.3 \pm 5.4$ & $-1.7 \pm 6.9$ & -9.2 & $251.1^{\mathrm{a}}$ \\
\hline & & & & & & & $252.0 \pm 10.5$ \\
\hline
\end{tabular}

Using $D_{0}^{0}\left(\mathrm{Ge}_{2}\right)=260.4 \pm 7.0 \mathrm{~kJ} \mathrm{~mol}^{-1}$, from Ref. 8. 
(1), by averaging the second- and third-law enthalpies. The average of the second- and third-law enthalpies for reaction (2), plus the knowledge that $D_{0}^{0}\left(\mathrm{Ge}_{2}\right)=260.4 \pm 7.0$ $\left(D_{298}^{0}=263.5\right) \mathrm{kJ} \mathrm{mol}^{-18}$ yielded a dissociation energy for PdGe of $D_{0}^{0}=251.1 \pm 8.8\left(D_{298}^{o}=253.0\right) \mathrm{kJ} \mathrm{mol}^{-1}$. The selected dissociation energy of $\mathrm{PdGe}(\mathrm{g}) D_{0}^{\circ}=252.0 \pm 10.5$ $\left(D_{298}^{0}=254.7\right) \mathrm{kJ} \mathrm{mol}^{-1}$ was taken as the average dissociation energy for reactions ( 1 ) and (2). The above thermochemical results are summarized in Table VIII. The heats of formation of $\operatorname{PdGe}(\mathrm{g})$ were calculated as $\Delta H_{f, 0}^{0}$ $=494.8 \pm 7.2$ and $\Delta H_{f, 298}^{0}=496.4 \mathrm{~kJ} \mathrm{~mol}^{-1}$, using the heat of vaporization ${ }^{19}$ of $\mathrm{Pd}(\mathrm{g})$ as $\Delta H_{v, 0}^{0}=375.7 \pm 2.1$ and $\Delta H_{v, 298}^{0}=376.6 \mathrm{~kJ} \mathrm{~mol}^{-1}$ and of $\mathrm{Ge}(\mathrm{g})$ as $\Delta H_{v, 0}^{0}$ $=371.7 \pm 2.1$ and $\Delta H_{v, 298}^{0}=374.5 \mathrm{~kJ} \mathrm{~mol}^{-1}$.

\section{CONCLUSIONS}

In the present work we have presented results of both theoretical and experimental investigations of the gaseous molecule PdGe. The electronic structure and the nature of bonding of the molecule have been elucidated through all electron $a b$ initio $\mathrm{HF}-\mathrm{Cl}$ calculations. The electronic states and the molecular constants resulting from our theoretical work have been utilized to derive the Gibbs energy functions for the PdGe molecule needed to determine the dissociation energy from the Knudsen cell mass spectrometric data.

The experimental dissociation energy of the PdGe molecule as determined in the present work is $D_{0}^{0}=252.0 \pm 10.5$ $\left(D_{298}^{0}=254.7\right) \mathrm{kJ} \mathrm{mol}^{-1}$. This value is comparable to the dissociation energy of $256.6 \pm 12 \mathrm{~kJ} \mathrm{~mol}^{-1}$ reported in a correspondence by Peeters et al., ${ }^{9}$ although the earlier literature value reduces to $245.1 \mathrm{~kJ} \mathrm{~mol}^{-1}$ after correction for the thermal functions of PdGe and $\mathrm{Ge}_{2}$ molecules used in our work.

In our theoretical work we have especially described the interaction between a Pd atom in its ${ }^{1} S(4 d)^{10}$ ground term and a Ge atom in the $(4 s)^{2}(4 p)^{2}$ configuration. From the calculated results we predict that the PdGe molecule has a ${ }^{3} \Pi$ ground state with two low-lying excited states ${ }^{3} \Sigma^{-}$and ${ }^{1} \Sigma^{+}$. The electronic structure of the PdGe molecule is consistent with a simple molecular orbital picture in which the $17 \sigma$ orbital is slightly below the $9 \pi$ orbital. The $17 \sigma$ orbital is essentially the $4 p \sigma$ of Ge while the $9 \pi$ orbital is the Ge $4 p \pi$.

The chemical bond in the PdGe molecule can be interpreted in terms of donation and backdonation of charge. From the deformation density map (Fig. 3 ) it is evident that the bond is polar with a small charge transfer from the Pd to the $\mathrm{Ge}$ atom.

\section{ACKNOWLEDGMENTS}

The computations have been performed at the Computing Service Center at Texas A\&M University and at UNI $\cdot C$, the Technical University of Denmark. The computational work in Denmark has been supported by the Danish Natural Science Research Council. I.S. acknowledges the Royal Danish Academy of Sciences and Letters for awarding the Niels Bohr fellowship. The work at Texas A\&M University has been supported by the National Science Foundation under Grant No. CHE-8219476, and the Robert A. Welch Foundation under Grant No. A-387. The authors also appreciate the support by a NATO Grant No. RG. $85 / 0448$ for international collaboration in research.

'Faraday Symposia of the Royal Society of Chemistry No. 14, Diatomic Metals and Metallic Clusters (1980).

${ }^{2}$ Metal Bonding and Interactions in High Temperature Systems, edited by J. L. Gole and W. C. Stwalley, Am. Chem. Soc. Symp. Ser. 178 (American Chemical Society, Washington, D.C., 1982).

${ }^{3}$ I. Shim and K. A. Gingerich, J. Chem. Phys. 76, 3833 (1982).

${ }^{4}$ I. Shim and K. A. Gingerich, J. Chem. Phys. 81, 5937 (1984).

${ }^{5}$ I. Shim and K. A. Gingerich, Surf. Sci. 156, 623 (1985).

${ }^{6}$ I. Shim and K. A. Gingerich, J. Chem. Phys. 80, 5107 (1984).

${ }^{7}$ I. Shim, H. M. Nagarathna-Naik, and K. A. Gingerich, Int. J. Quantum Chem. 29, 975 (1986).

${ }^{8}$ J. E. Kingcade, H. M. Nagarathna-Naik, I. Shim, and K. A. Gingerich, J. Phys. Chem. 90, 2830 (1986).

${ }^{9}$ R. Peeters, A. M. Vander Auwera-Mahieu, and J. Drowart, Z. Naturforsch. Teil A 26, 327 (1971).

${ }^{10}$ C. C. J. Roothaan, Rev. Mod. Phys. 32, 179 (1960)

"J. Almlöf, in Proceedings of the second Seminar on Computational Problems in Quantum Chemistry (Max Planck Institut, München, 1973).

${ }^{12}$ The ALCHEMY program system has been written at IBM Research Laboratory in San Jose, CA by P. S. Bagus, B. Liu, M. Yoshimine, and A. D. McLean.

${ }^{13}$ C. R. Sarma and S. Rettrup, Theor. Chim. Acta (Berlin) 46, 63 (1977); S. Rettrup and C. R. Sarma, ibid. 46, 73 (1977).

${ }^{14} \mathrm{H}$. Johansen (private communication).

${ }^{15}$ S. Huzinaga, J. Chem. Phys. 66, 4245 (1977).

${ }^{16}$ I. Shim and K. A. Gingerich (to be published).

${ }^{17}$ E. A. Ballik and D. A. Ramsay, Astrophys. J. 137, 61 (1963); 137, 84 (1963).

${ }^{18}$ G. Pacchioni, Mol. Phys. 49, 727 (1983).

${ }^{19}$ R. Hultgren, P. J. Desai, D. T. Hawkins, M. Gleiser, K. K. Kelly, and D. D. Wagman, Selected Values of Thermodynamic Properties of the Elements (American Society for Metals, Metal Park, OH 1973).

${ }^{20} \mathrm{D}$. R. Stull and H. Prophet, in The Characterization of High Temperature Vapors, edited by J. L. Margrave (Wiley-Interscience, New York, 1967), pp. $359-424$. 